\title{
MiniWall Tool for Analyzing CFD and Wind Tunnel Large Data Sets
}

\author{
Michael J. Schuh ${ }^{1}$ and John E. Melton ${ }^{1}$ \\ NASA Ames Research Center, Moffett Field, CA, 94035, USA \\ and \\ Paul M. Stremel ${ }^{2}$ \\ Science and Technology Corporation, NASA Research Park, CA, 94035, USA
}

\begin{abstract}
It is challenging to review and assimilate large data sets created by Computational Fluid Dynamics (CFD) simulations and wind tunnel tests. Over the past 10 years, NASA Ames Research Center has developed and refined a software tool dubbed the "MiniWall" to increase productivity in reviewing and understanding large CFD-generated data sets. Under the recent NASA ERA project, the application of the tool expanded to enable rapid comparison of experimental and computational data. The MiniWall software is browser based so that it runs on any computer or device that can display a web page. It can also be used remotely and securely by using web server software such as the Apache HTTP Server. The MiniWall software has recently been rewritten and enhanced to make it even easier for analysts to review large data sets and extract knowledge and understanding from these data sets. This paper describes the MiniWall software and demonstrates how the different features are used to review and assimilate large data sets.
\end{abstract}

$\begin{array}{ll}\text { Prat } & \begin{array}{c}\text { Nomenclature } \\ \text { Acronyms }\end{array} \\ \text { ARC } & =\text { Ames Research Center } \\ \text { CFD } & =\text { computational fluid dynamics } \\ \text { SLS } & =\text { Space Launch System }\end{array}$

\section{Introduction}

$\mathrm{T}$ he MiniWall software was inspired by the NASA Ames Research Center NAS hyperwall ${ }^{2}$. The hyperwall shown in Figure 1 is the second generation hyperwall created in $2008^{3}$. It is a 128 -screen tiled LCD wall in $8 \times 16$ configuration and is $23 \mathrm{ft}$ wide and $10 \mathrm{ft}$ high. The hyperwall helps researchers display, analyze, and study multidimensional datasets in meaningful ways, allowing the use of different tools, viewpoints, and parameters to display the same data or datasets. The MiniWall software was written by John Melton in 2006 to bring many of the benefits of the hyperwall to a researcher's desktop.

Modern CFD analyses and wind tunnel tests usually consist of very large run matrices as illustrated in Figure 2. Figure 3 shows an example of a folder view of the many images and plots that are often generated for each test condition. Comparing plots and images from different test conditions can quickly become a bewildering challenge when hundreds, and sometimes thousands, of data files are buried in numerous subdirectories. The MiniWall software was created to provide for easy and interactive data exploration. It allows the data to be displayed in the context of the test (i.e., Mach number versus Angle-of-Attack) and enables researchers to easily and quickly scan and view other related data. This capability greatly simplifies the task of extracting knowledge and understanding from what might otherwise be an overwhelming volume of data. Since the MiniWall simplifies the investigation and visualization of

\footnotetext{
${ }^{1}$ Aerospace Engineer, System Analysis Office, Aeronautics Directorate, Mail Stop 258-1.
}

${ }^{2}$ Senior Research Scientist, Mail Stop 258-1, AIAA Member.

American Institute of Aeronautics and Astronautics 


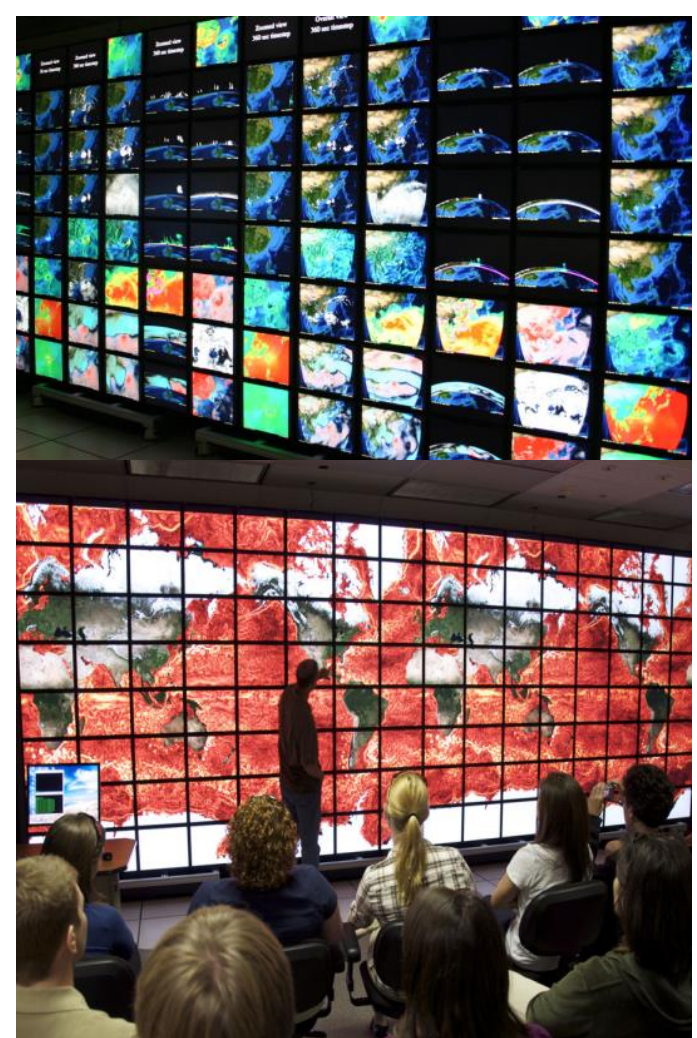

Figure 1. NASA hyperwall showing a scalable concurrent visualization of Typhoon Morakot from different perspectives at the same time (left) and a large simulation (right). large multi-file datasets, it naturally promotes the sharing of these results with collaborators who might be unfamiliar with or unable to search through the many files. Our experience with the MiniWall is that customers quickly request and adopt it after an initial exposure. They find the MiniWall easy to use and adapt for understanding their own simulations and experiments.

$\begin{array}{lccccc}\text { Configuration } & \begin{array}{c}\text { Angle ofSide Slip } \\ \text { Attack }\end{array} & \text { Angle } & & & \\ & & & & & \\ \text { Baseline-aircraft } & 0 & 0 & 0.75 & 45 & \text { High } \\ \text { Baseline-aircraft } & 2 & 0 & 0.75 & 45 & \text { High } \\ \text { Baseline-aircraft } & 4 & 0 & 0.75 & 45 & \text { High } \\ \text { Baseline-aircraft } & 8 & 0 & 0.75 & 45 & \text { High } \\ \text { Baseline-aircraft } & 0 & 0 & 0.25 & 0 & \text { Med } \\ \text { Baseline-aircraft } & 4 & 0 & 0.25 & 0 & \text { Med } \\ \text { Baseline-aircraft } & 6 & 0 & 0.25 & 0 & \text { Med } \\ \text { Baseline-aircraft } & 8 & 0 & 0.25 & 0 & \text { Med } \\ \text { Baseline-aircraft } & 0 & 5 & 0.25 & 0 & \text { Low } \\ \text { Baseline-aircraft } & 6 & 5 & 0.25 & 0 & \text { Low } \\ \text { Baseline-aircraft } & 0 & 10 & 0.25 & 0 & \text { Low } \\ \text { Baseline-aircraft } & 6 & 10 & 0.25 & 0 & \text { Low } \\ & & & & & \\ \text { Modified-aircraft } & 0 & 0 & 0.75 & 45 & \text { High } \\ \text { Modified-aircraft } & 2 & 0 & 0.75 & 45 & \text { High } \\ \text { Modified-aircraft } & 4 & 0 & 0.75 & 45 & \text { High } \\ \text { Modified-aircraft } & 8 & 0 & 0.75 & 45 & \text { High }\end{array}$

Figure 2. Example CFD and wind tunnel test matrix.

The MiniWall software was originally written by John Melton using a combination of HTML, JavaScript, and Microsoft's ActiveX software. Paul Stremel rewrote it using modern JavaScript and HTML standards which enabled it to be run on non-Microsoft Windows-based computers. Michael Schuh recently rewrote it using JavaScript objects and extended the MiniWall software to have additional functionality that easily accommodates different usages. While it currently works best with the Google Chrome browser, it will soon be updated to run well under additional browsers such as Firefox, Internet Explorer, and Microsoft Edge.

The MiniWall software provides three different views of the data - the main image table, the multi-image window, and the single image window. There are many features that enable an analyst to move through the solution matrix, create different views of the results, and zoom in and out on the images to view the results in detail.

The solution images are typically saved in jpeg or png formats but can be saved in any format that can be displayed by a browser. The images are accessed by the MiniWall software using a structured file name convention. For example, "_Mach0.9_" and "_Mach1.2_" can be used in the filename to specify different Mach numbers. Utilizing this structure enables the software to fill the MiniWall views with the desired data. Wind tunnel results, CFD data, and other data sets can be presented and analyzed using the MiniWall.

The MiniWall can be used by accessing the HTML, JavaScript, and image files that make up the MiniWall from a local file system or through a web server such as Apache HTTP Server or Nginx. Web access can be protected by standard security systems such as using https and requiring a valid username and password for access. 
This paper provides an overview of the MiniWall software and some details on the three views of the data and how to work with them. Many of the features were added as a result of intensely using the software, identifying opportunities to make it more useful, and adding those features. It is easy to use to view data and gain understanding and knowledge from large data sets. While extensive programing experience is not necessary to configure the MiniWall software, it does help if one has some basic programing skills to set up a MiniWall for a new data set. A modestly skilled programmer should be able to extend the software to provide custom functionality.

\section{Overview - Three Views of the Data}

There are three different ways to use the MiniWall to view images. Figure 4 shows the main image table for the MiniWall. The images shown are from CFD simulations of a honeycomb vent design analyzed for the Space Launch System (SLS) rocket. This is the main view of the images and is what the user sees when they first open a MiniWall to view a data set. The images in Figure 4 show computed Mach number contours for three different vent diameters in the vertical axis and three different pressure ratios between the supply pressure and freestream pressure in the horizontal axis. In this example, the main image table is made up of pressure ratio versus geometry. The MiniWall can be configured to have any combination of parameters such as angle of attack versus beta, Mach, or results from different wind tunnel tests and CFD codes. This is all part of the flexibility and extensibility of the MiniWall software.

The multi-image window shown in Figure 5 shows the first thirty images available for a particular vent configuration selected from the main image table shown in Figure 4. The multi-image window is opened by "shift alt left-mouse" clicking on an image in the main image table. The multi-image window view of the data is used to show all of the images available for a data set in the main image table. The rest of the available images in the multi-image window are brought into view by scrolling the multi-image window. By "shift left-mouse" clicking on an image in either the main image table or the multi-image window, a single-image window like the one shown in Figure 6 will open showing a larger view of the image clicked on. This is the third way of viewing the data and is used to look at particular images in detail. The software is written to easily coordinate what is shown in these three views of the data. The help screen shown in Figure 7 is brought up by typing the " $h$ " key in any of the three views of the data. The help screen gives a brief description of how to use the MiniWall. With the help screen and this paper, a new user should be able to figure out how to use all of the features of the software.

The next sections describe these three data views in more detail.

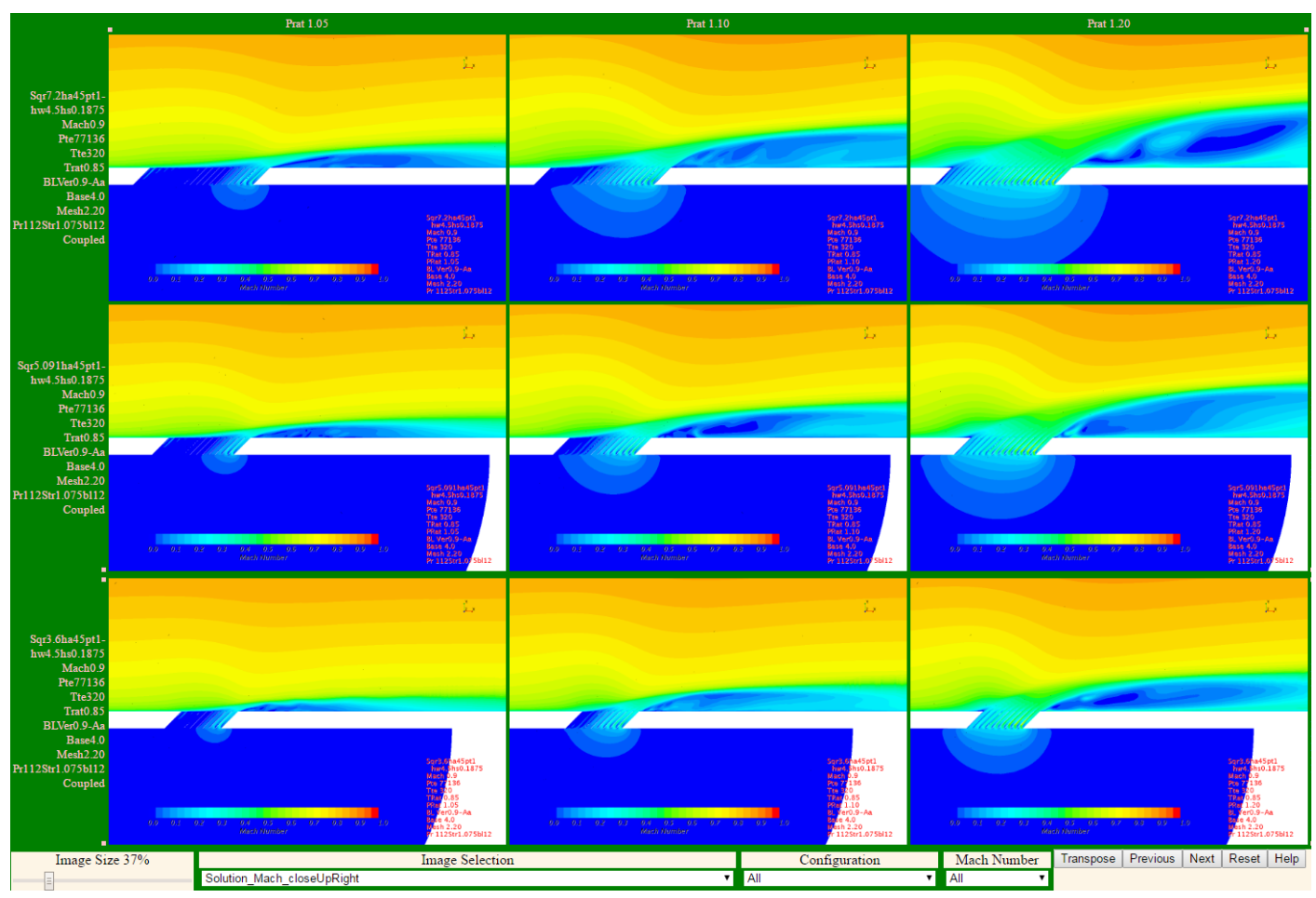

Figure 4. MiniWall main image table with CFD solutions for three different sized Space Launch Systems (SLS) honeycomb vents with three different pressure ratios. 


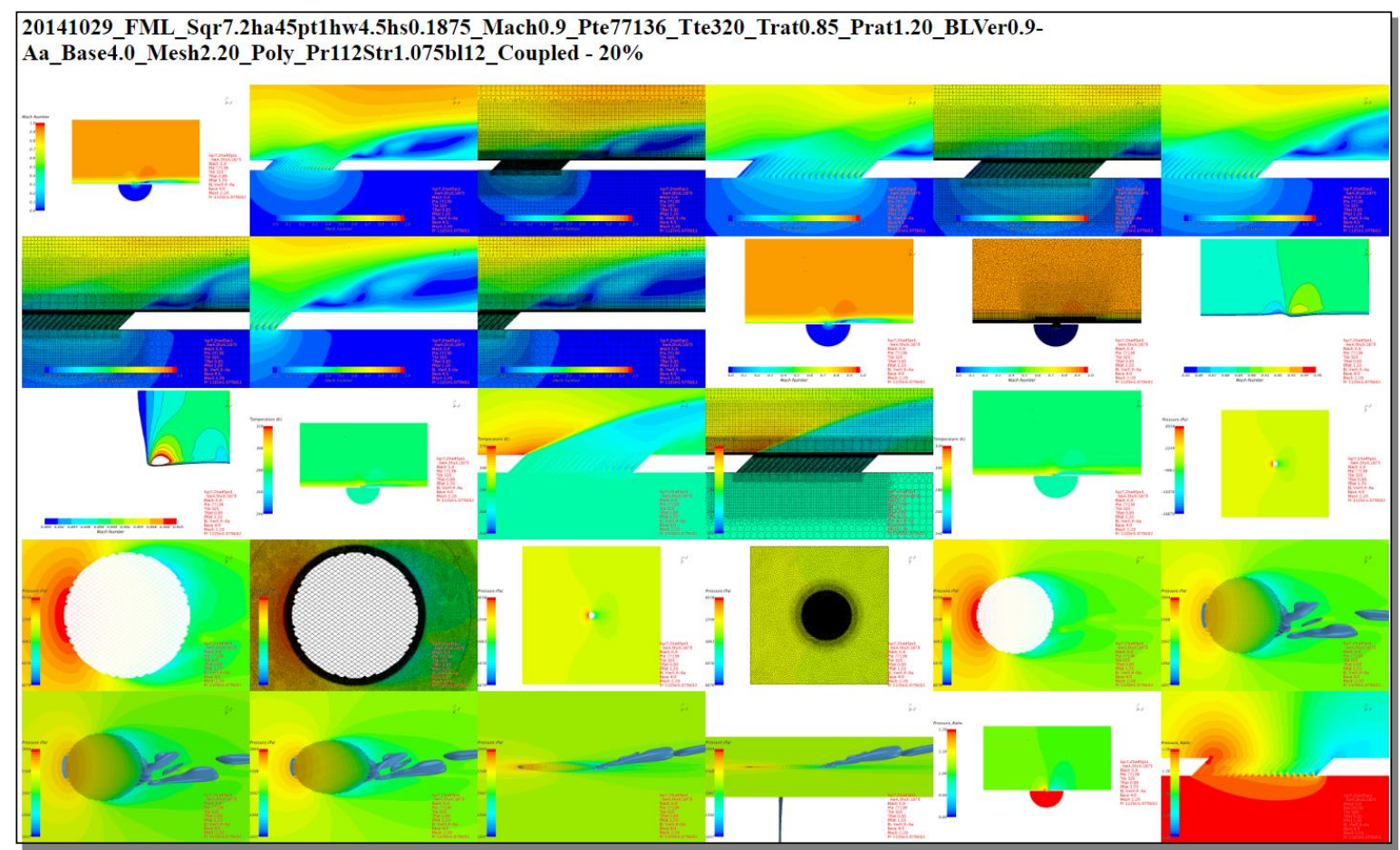

Figure 5. MiniWall multi-image window showing solution images available for a selected SLS honeycomb vent configuration.

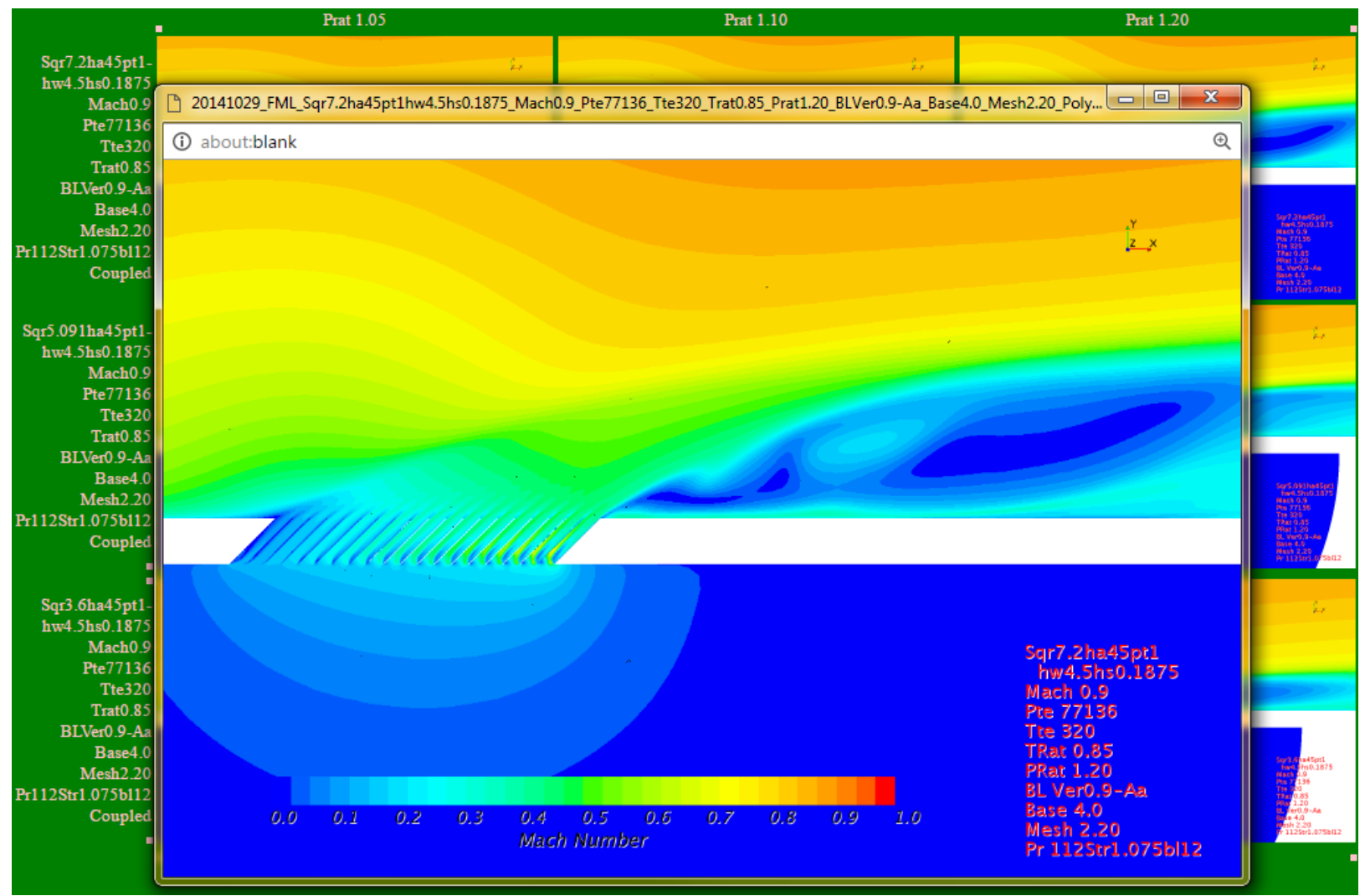

Figure 6. MiniWall single-image window showing enlarged view of a solution image opened on top of the main image table. 


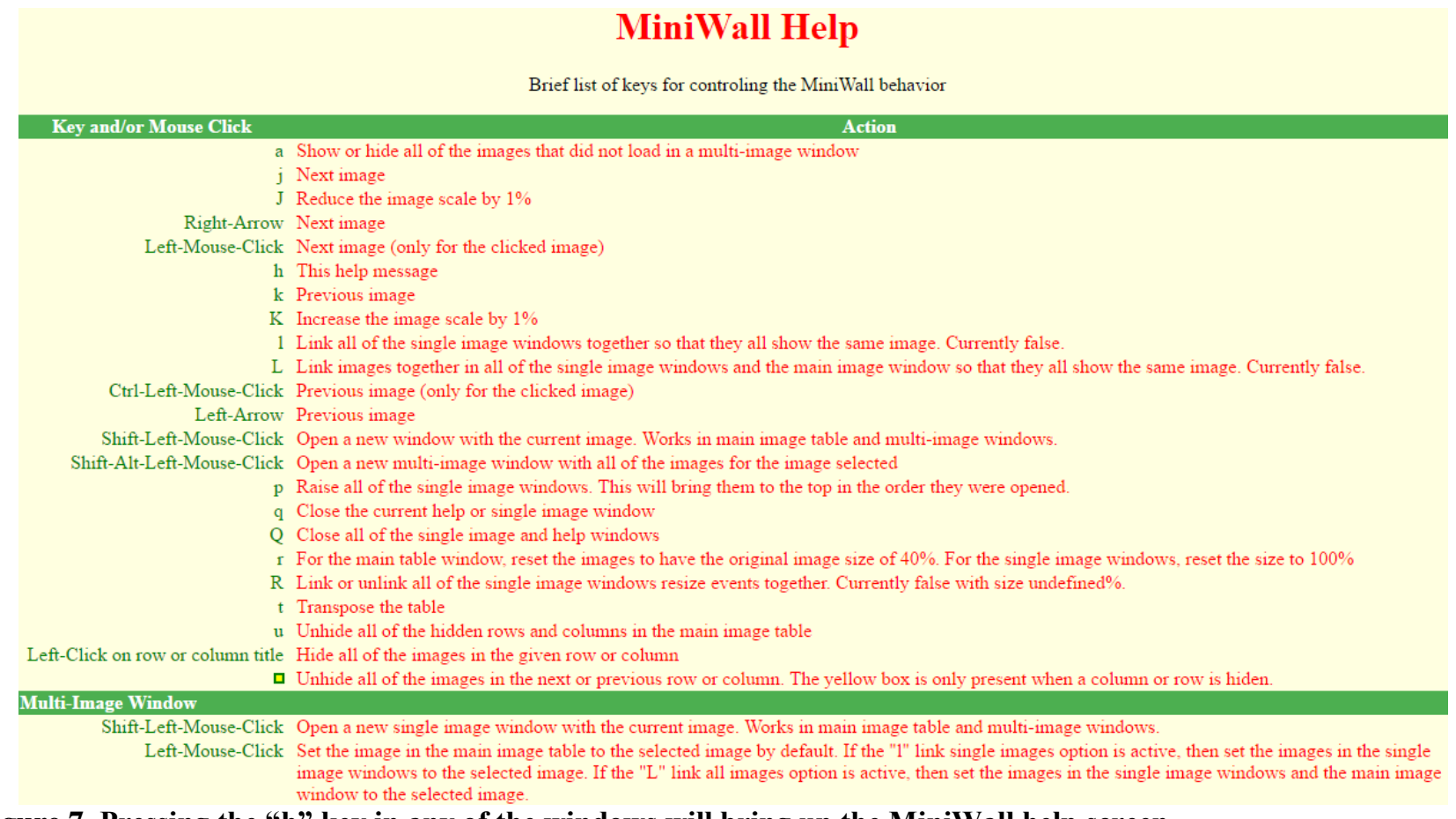

Figure 7. Pressing the "h" key in any of the windows will bring up the MiniWall help screen.

\section{Main Image Table}

The main image table view shows all of the available solutions. Sparse data sets as shown in Figure 8 are handled without issue. Missing images are replaced by red cells with text showing the current image name. There are several controls at the bottom of the main image table view as shown in Figure 8. Moving the slider in the "Image size" control changes the size of all of the images in the main image window. Pressing the "J" and " $\mathrm{K}$ " keys in the main image window reduces and increases the size of the images by $1 \%$ respectively. The "Image Selection" control is used to change the images shown in the table. The images can also be changed by left-mouse clicking on the "Next" and "Previous" buttons in the lower right corner of the main image window. Some will find that it is much easier to change the images by pressing the " $\mathrm{j}$ " and " $\mathrm{k}$ " keys. Doing so will go to the next and previous images for the " $\mathrm{j}$ " and " $\mathrm{k}$ " keys respectively. The " $\mathrm{j}$ " and " $\mathrm{k}$ " are two of the home keys for the right hand on a typical keyboard. They are used in a similar way in the "vi" editor. "Left-mouse" clicking on an image will change the image in that table cell to the next image in the "Image Selection" list each time it is clicked. "Ctrl-left-mouse" clicking on an image will change the image to the previous image in the list each time it is clicked. The "Configuration" control is used to select a subset of the images to look at. For example, Figure 8 shows three different honeycomb cell types and an "All" option to show all of the types. Other specialized controls can be added such as the "Mach Number" control with options "All", "0.9", and "1.4" as shown in Figure 8. The "Transpose" button is used to transpose the main image table. This is helpful for rearranging images that were in a column into rows across several side-by-side monitors all at once. The "Reset" button resets the size of the images in the main image table to the initial size. The initial size is configurable in the HTML that sets up the main image table.

It is helpful sometimes to hide columns and or rows of images in the main image window. This enables the investigator to compare data sets that are separated by one or more rows. Columns and rows are hidden by "leftmouse" clicking on the column or row title text. Hidden rows and columns are indicated by small pink squares at the edges of the rows and columns as shown in Figure 9. Clicking on these small pink squares will unhide a row or a column. Pressing the "u" key unhides all rows and columns.

The main image table is created by opening an HTML file similar to the "miniwall_vent.html" file shown in Figure 10. The HTML loads two JavaScript files that contain the code for making the MiniW $\bar{W}$ all and specifying the data sets and control options in the main image window. Lines 16 through 22 specify some defaults and line 25 creates the main image table using the JavaScript in the "utils_object.js" file. JavaScript code from "setup_vent_object.js" file is shown in Figure 11 and it shows how the main image table data sets and the control options are specified. Some of the control values are omitted in Figure 11 and are replaced by the text "[lines not shown]". This was done to make the contents more readable. The "utils_object.js" file is about two thousand lines long and is responsible for creating the different

American Institute of Aeronautics and Astronautics 
data views and communicating between them. Most users will not need to do anything in the "utils_object.js" file. All they will need to do is update the "miniwall_vent.html" and "utils_object.js" files to set up the MiniWall for a new data set.

"Shift-left-mouse" or "Shift-Alt-left-mouse" clicking on any image in the main image table will respectively bring up a single image window for the image clicked or a multi-image window for all of the images in the data set for the image that was clicked. These views of the data will be described in the next two sections.

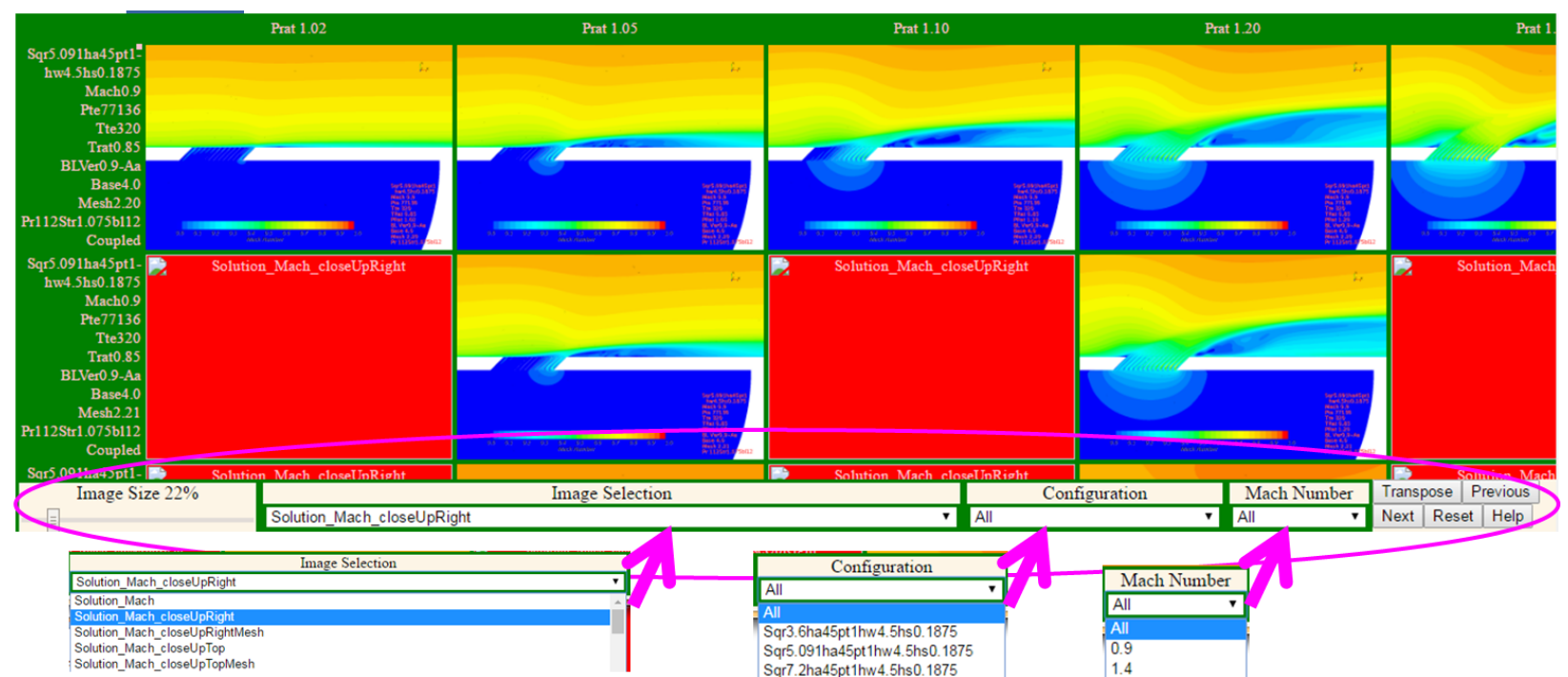

Figure 8. MiniWall main image table showing red cell for missing data and controls at the bottom of the main image table view.

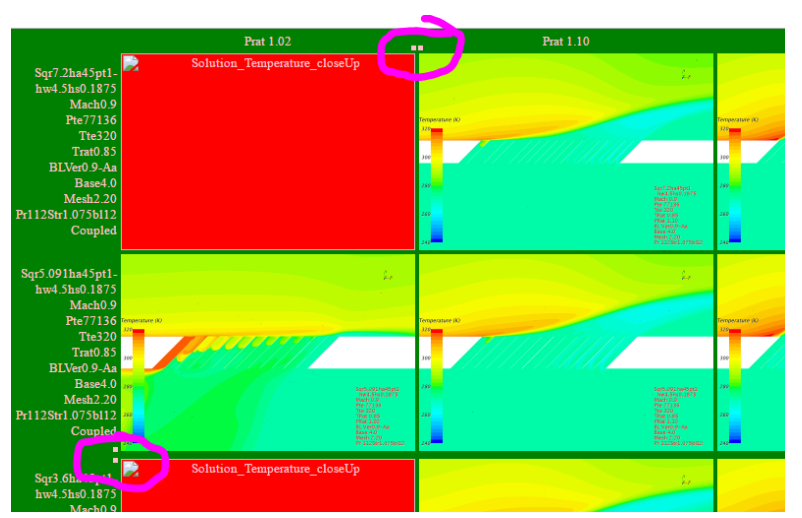

Figure 9. Hidden rows and columns are marked by small pink boxes at the edges of the rows and columns.

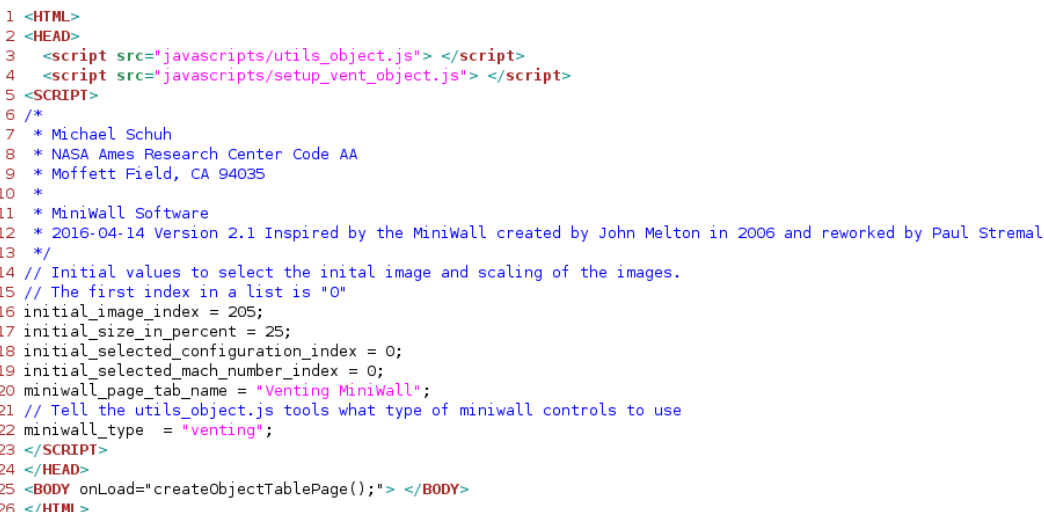

Figure 10. HTML used to create and define the MiniWall. 


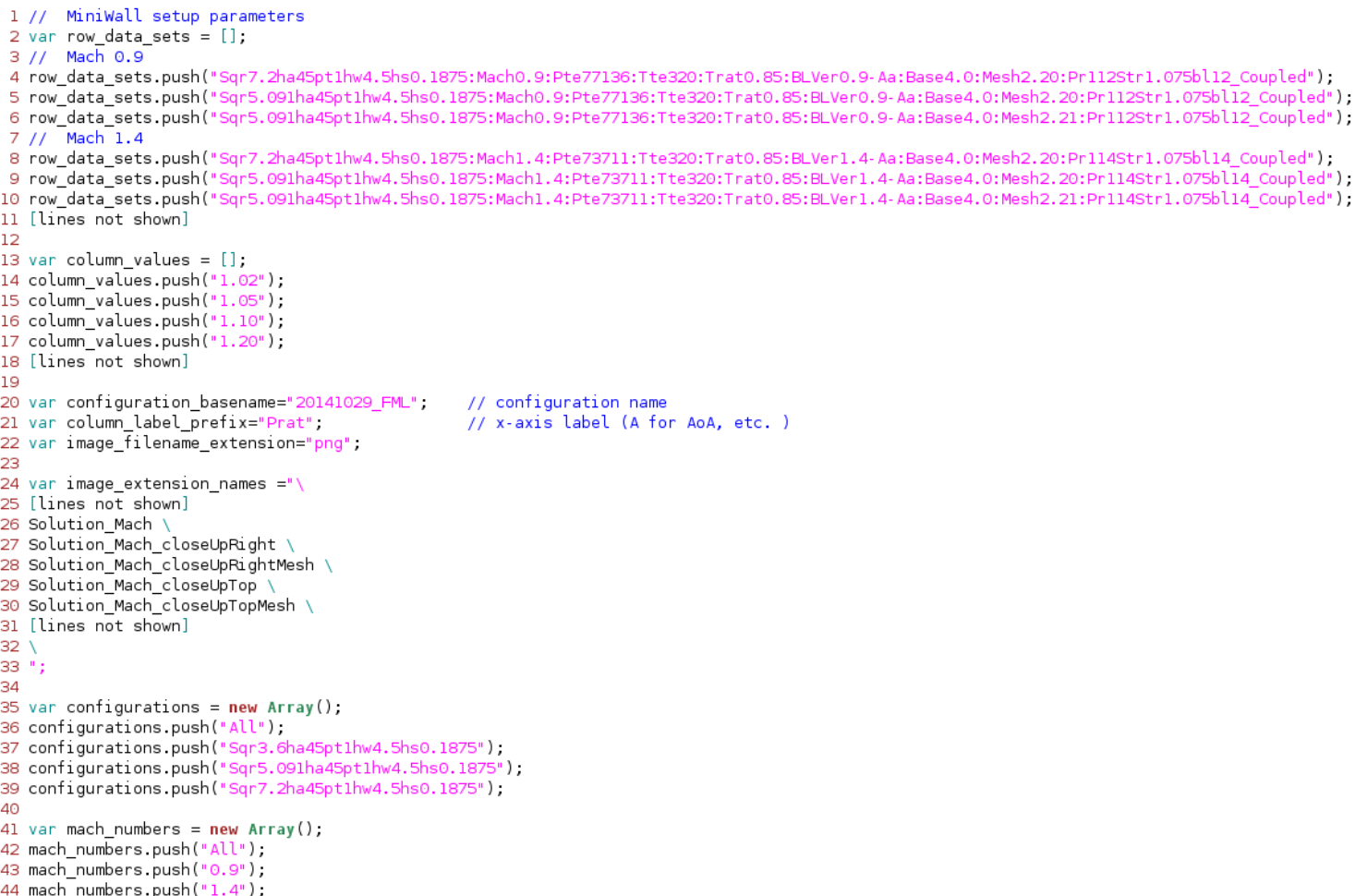

Figure 11. JavaScript used to configure the MiniWall main image window.

\section{Multi-Image Window}

The multi-image window shows all of the images that are available for a data set. The multi-image window is opened by "shift left-mouse" clicking on an image in the main image table. The multi-image window shown in Figure 5 shows an example of the many images available for a data set represented by a cell in the main image table. The main image table "Image Selection" control has a full list of all of the images that can be shown. The multi-image window opens showing the available images. Sometimes it is helpful to see which, if any, images are not available for a data set. Pressing the "a" key in the multi-image window will display red cells with the missing image name in text. This is shown in Figure 12. Clicking on an image in the multi-image window changes every image in the main image table to that image. This makes the multi-image window very useful for selecting which images to display. Clicking on the "q" key in the multi-image window will "quit" and close that window. "Shift-left-mouse" clicking on an image in the multi-image window will open that image in a single image window. "Shift-alt-left-mouse" clicking on several images in the main image table will open a multi-image window for each image clicked.

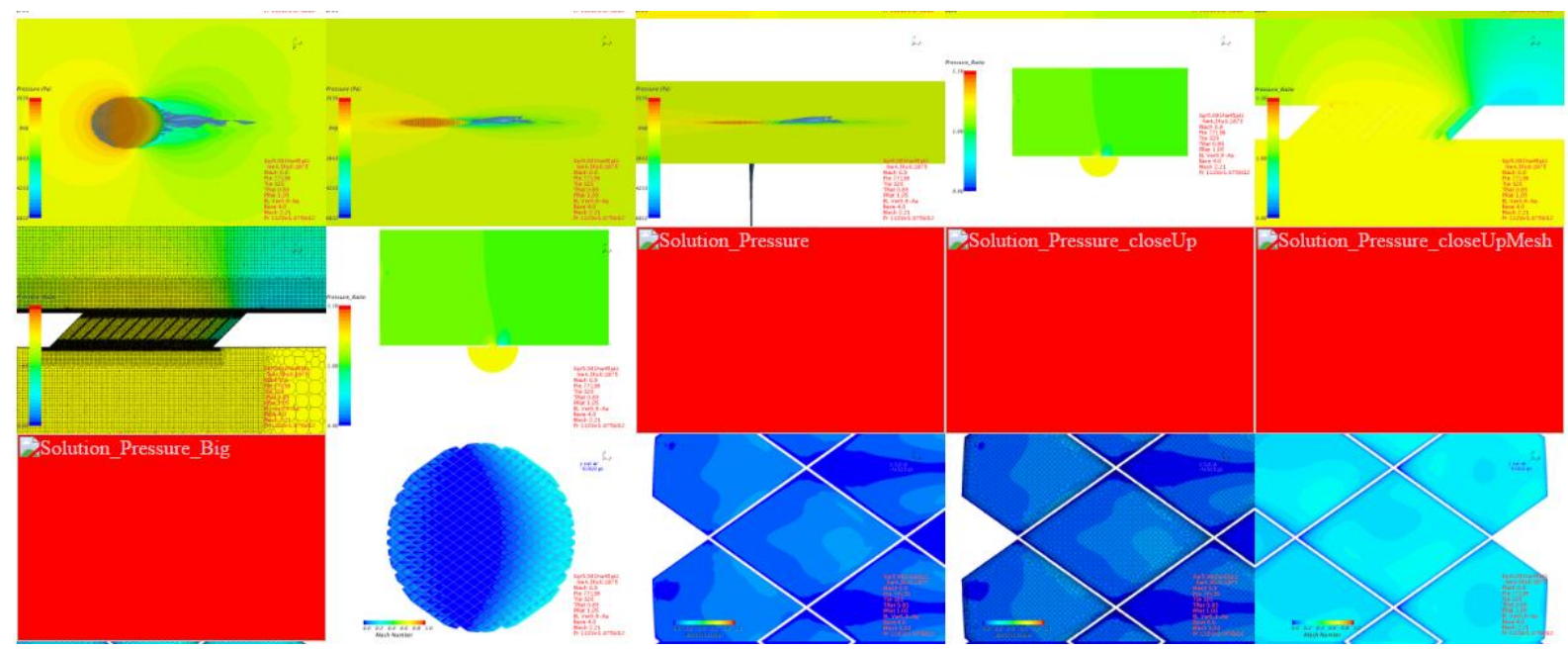

Figure 12. Multi-image window with missing images shown in red with missing image name text.

American Institute of Aeronautics and Astronautics 


\section{Single-Image Window}

It is often useful to open up an image from the main image table or multi-image window and view it at a larger size. An example of this is shown in Figure 6. Multiple single images can be open for the same or different data sets. The image displayed in the single image window can be changed by clicking on it or with keyboard commands. As with the main image table, "Left-mouse" clicking on the image or entering the "j" key will change the image to the next available image in the "Image Selection" control list. "Shift-left-mouse" clicking on the image or entering the " $\mathrm{k}$ " changes the image to the previous image available. Unavailable images are skipped and the next and previous image requests will wrap to the beginning or the end of the list. The size of the images can be changed by using the mouse to resize the single image window or by using the " $\mathrm{J}$ " and " $\mathrm{K}$ " keys to reduce or enlarge the image by $1 \%$ respectively.

There are several keyboard commands that make working with single image windows easier and more productive. The "l" key can be used to toggle a "l-link" setting so that all images in single image windows show the same image. When the "l-link" toggle is set, all single image windows change to the same image whenever a "next" or "previous" image is requested via the keyboard or mouse inputs. With the "l-link" image toggle set, clicking on an image in the multi-image window sets all single image windows to that image. Setting the "l-link" image toggle unlinks the connection between clicking on images in the multi-image window and setting the images in the main image table. All images in the single image windows and the main image table can be linked together by pressing the "L" in any of the single image windows, multi-image windows, or the main image table. This way all images change together when a "next" or "previous" image is requested in any of the windows or whenever an image is "left-mouse" clicked in the multi-image window. The state of the "l-link" and "L-link" toggles can be checked by opening a new help window using the "h" key.

Single image windows can also be linked to have the same size and change to the same size when a single image window is resized. This is done by using the " $R$ " key to link the window "Resize" events together. Hitting an "r" key in a single image window will reset that window size to $100 \%$ of the image size.

As single image windows are opened by "shift-left-mouse" clicking on images in the main image table or the multi-image window, previously opened single image windows sometimes end up below the main image table and are a nuisance to bring to the top using the windows manager. The "p" key can be used in a single image window to "pop" all single image windows to the top of all other windows. Another useful key is the "Q" key which closes all open single image and help windows. The "q" key will close the currently active single image window or help window.

\section{Summary and Conclusion}

The MiniWall is a great tool for looking through large data sets and comparing different results with each other. It can be configured to work with wind tunnel data, CFD data, and other data sets. As long as the data can be presented in a browser, the MiniWall can be configured to view it. This makes the MiniWall a valuable tool for viewing, understanding, and extracting knowledge from large complex data sets. Future planned enhancements include the interactive display of 3D datasets using WebGL and related tools. The ability to use standard browsers to run the MiniWall software from desktop computers, laptops, and handheld devices either locally or over networks adds to this value.

\section{Acknowledgements}

The MiniWall software was written and used to support many projects through the years. Most of the recent refactoring and enhancements to the code were made while supporting the NASA Integrated Aviation Systems Research Program's Environmentally Responsible Aviation Project.

\section{References}

\footnotetext{
${ }^{1}$ Space Launch System Overview website page, https://www.nasa.gov/exploration/systems/sls/overview.html [retrieved 2 Dec 2016].

${ }^{2}$ NASA Ames High-End Computing Capability website page, https://www.nas.nasa.gov/hecc/resources/viz_systems.html [retrieved 5 Dec 2016].

${ }^{3}$ Biegel, B. A., Sandstrom, T. A., Henze, C., and Levit, C, "The Hyperwall," CMV 2003 Conference, London, United Kingdom, July 2003.
} 